\title{
ALGEBRAIC AND ANALYTIC PROPERTIES OF SEMIGROUPS RELATED TO FIXED POINT PROPERTIES OF NON-EXPANSIVE MAPPINGS
}

\author{
ANTHONY TO-MING LAU † AND YONG ZHANG $\ddagger$
}

\begin{abstract}
The purpose of this paper is to give an updated survey on various algebraic and analytic properties of semigroups related to fixed point properties of semigroup actions on a non-empty closed convex subset of a Banach space or, more generally, a locally convex topological vector space.
\end{abstract}

Dedicated to Professor Karl H. Hofmann with admiration and respect

\section{INTRODUCTION}

In this paper we shall present an updated survey on fixed point properties of a semigroup of non-expansive mappings on a closed convex subset of a Banach space (or more generally, a locally convex topological space) related to certain algebraic and analytic properties of the semigroup. We begin with some historic developments of the investigation.

Let $E$ be a Banach space and let $K$ be a non-empty bounded closed convex subset of $E$. We say that $K$ has the fixed point property if for every non-expansive mapping $T: K \rightarrow K$ (i.e. $\|T x-T y\| \leq\|x-y\|$, $x, y \in K), K$ contains a fixed point for $T$.

It follows from Bruck [8] that if $E$ is a Banach space with the weak fixed point property (i.e. any weakly compact convex subset of $E$ has the fixed point property), then any weakly compact convex subset $K$ of $E$ has the (common) fixed point property for any commutative semigroup acting on $K$.

A well-known result of Browder [7] asserts that if $E$ is uniformly convex, then $E$ has the weak fixed point property. Kirk 34] extended

1991 Mathematics Subject Classification. Primary 43A07 Secondary 43A60, 22D05 46B20.

Key words and phrases. amenability properties, semigroups, nonexpansive mappings, weakly compact, weak* compact, convex sets, common fixed point, invariant mean, submean.

$\dagger$ Supported by NSERC Grant ZC912.

$\ddagger$ Supported by NSERC Grant 1280813 . 
this result by showing that if $K$ is a weakly compact subset of $E$ with normal structure, then $K$ has the fixed point property. Other examples of Banach spaces with the weak fixed point property include $c_{0}, \ell^{1}$, trace class operators on a Hilbert space and the Fourier algebra of a compact group (see [13, 21, 45, 48, 49, 60, 62, 64, 77] and [5, 6] for more details). However, as shown by Alspach [1], $L^{1}[0,1]$ does not have the weak fixed point property.

Given a semigroup $S$, we let $\ell^{\infty}(S)$ be the $\mathrm{C}^{*}$-algebra of bounded complex-valued functions on $S$ with the supremum norm and pointwise multiplication. For each $a \in S$ and $f \in \ell^{\infty}(S)$ let $\ell_{a} f$ and $r_{a} f$ be the left and right translates of $f$ by $a$, respectively; i.e. $\ell_{a} f(s)=f(a s)$ and $r_{a} f(s)=f(s a)(s \in S)$. Let $X$ be a closed subspace of $\ell^{\infty}(S)$ containing constants and invariant under translations. Then a linear functional $m \in X^{*}$ is called a mean if $\|m\|=m(1)=1 ; m$ is called a left (resp. right) invariant mean, denoted by LIM (resp. RIM), if $m\left(\ell_{a} f\right)=m(f)$ (resp. $\left.m\left(r_{a} f\right)=m(f)\right)$ for all $a \in S, f \in X$. A discrete semigroup $S$ is left (resp. right) amenable if $\ell^{\infty}(S)$ has a LIM (resp. RIM). Let X be a $\mathrm{C}^{*}$-subalgebra of $\ell^{\infty}(S)$. Then the spectrum of $\mathrm{X}$ is the set of non-zero multiplicative linear functionals on $\mathrm{X}$ equipped with the relative weak* topology. Every $s \in S$ is a multiplicative linear functional on $\mathrm{X}$ if we regard it as the evaluation functional: $\langle s, f\rangle=f(s)$ for $f \in X$.

Let $S$ be a semitopological semigroup, i.e. $S$ is a semigroup with Hausdorff topology such that for each $a \in S$, the mappings $s \mapsto s a$ and $s \mapsto$ as from $S$ into $S$ are continuous. We let $C_{b}(S)$ be the space of all bounded continuous complex-valued functions on $S$. We denote by $L U C(S)$ the space of all left uniformly continuous functions on $S$, i.e. all $f \in C_{b}(S)$ such that the mapping $s \mapsto \ell_{s} f$ : $S \rightarrow C_{b}(S)$ is continuous. The semitopological semigroup $S$ is called left amenable if $L U C(S)$ has a LIM. We note that if $S$ is discrete, then $L U C(S)=C_{b}(S)=\ell^{\infty}(S)$ and so left amenability of $S$ coincides with that previously defined. Now denote by $A P(S)$ the space of all $f \in C_{b}(S)$ such that $\mathcal{L O}(f)=\left\{\ell_{s} f: s \in S\right\}$ is relatively compact in the norm topology of $C_{b}(S)$, and denote by $W A P(S)$ the space of all $f \in C_{b}(S)$ such that $\mathcal{L O}(f)$ is relatively compact in the weak topology of $C_{b}(S)$. Functions in $A P(S)$ (resp. $W A P(S)$ ) are called almost periodic (resp. weakly almost periodic) functions on $S$. Later in this paper we will also need to consider the set $\mathcal{R O}(f)=\left\{r_{s} f: s \in S\right\}$. As well known, $f \in A P(S)$ (resp. $f \in W A P(S)$ ) if and only if $\mathcal{R O}(f)$ is relatively compact in the norm (resp. weak) topology of $C_{b}(S)$. Let $S^{a}$ (resp. $S^{w}$ ) be the almost periodic (resp. weakly almost periodic) compactification of $S$, i.e. $S^{a}$ (resp $S^{w}$ ) is the spectrum of the C*algebra $A P(S)$ (resp. $W A P(S)$ ). Then $S^{a}$ and $S^{w}$ are semitopological 
semigroups with multiplications defined by: $\langle m \cdot n, f\rangle=\langle m, n \cdot f\rangle$, where $n \cdot f(s)=\left\langle n, \ell_{s} f\right\rangle, m, n \in S^{a}$ (resp. $\left.S^{w}\right), f \in A P(G)$ (resp. $W A P(G))$. In fact, the multiplication in $S^{a}$ is even jointly continuous. In other words, $S^{a}$ is a topological semigroup.

The semitopological semigroup $S$ is called left reversible if any two closed right ideals of $S$ have non-void intersection, i.e. $\overline{a S} \cap \overline{b S} \neq \emptyset$ for any $a, b \in S$.

If $A$ is a subset of a topological space $E$, then $\bar{A}$ will denote the closure of $A$ in $E$. If in addition, $E$ is a linear topological space, then $[\overline{c o} A] \operatorname{co} A$ will denote the [closed] convex hull of $A$ in $E$.

An action of $S$ on a topological space $K$ is a mapping $\psi$ from $S \times K$ into $K$, denoted by $T_{s} x=\psi(s, x)(s \in S$ and $x \in K)$, such that $T_{s_{1} s_{2}} x=T_{s_{1}}\left(T_{s_{2}} x\right)\left(s_{1}, s_{2} \in S\right.$ and $\left.x \in K\right)$. The action is separately continuous or jointly continuous if the mapping $\psi$ is, respectively, separately or jointly continuous. For convenience, $T_{s} x$ is also denoted by $s x$, and we call $\mathcal{S}=\left\{T_{s}: s \in S\right\}$ a representation of $S$ on $K$.

When $K$ is a convex subset of a linear topological space, we say that an action of $S$ on $K$ is affine if for each $s \in S$, the mapping $x \mapsto s x: K \rightarrow K$ satisfies $s(\lambda x+(1-\lambda) y)=\lambda s x+(1-\lambda) s y$ for $s \in S$, $x, y \in K$ and $0 \leq \lambda \leq 1$. A locally convex topological space $E$ with the topology generated by a family $Q$ of seminorms will be denoted by $(E, Q)$. An action of $S$ on a subset $K \subseteq E$ is $Q$-non-expansive if $\rho(s \cdot x-s \cdot y) \leq \rho(x-y)$ for all $s \in S, x, y \in K$ and $\rho \in Q$.

The following fixed point property was proved by the first author [36, Theorem 4.1] (see also [65, 78]):

Theorem 1.1. Let $S$ be a semitopological semigroup. Then $A P(S)$, the space of continuous almost periodic functions on $S$, has a LIM if and only if $S$ has the following fixed point property:

(D): Whenever $S$ is a separately continuous and $Q$-non-expansive action on a compact convex subset $K$ of a separated locally convex space $(E, Q), K$ has a common fixed point for $S$.

It has been an open question for quite a long time (see [37, 40]) as to whether the existence of LIM on $W A P(S)$, the space of continuous weakly almost periodic functions on $S$, can be characterized by a fixed point property for non-expansive actions of $S$ on a weakly compact convex set.

It was proved by Hsu [28] (also see [52, Corollary 5.5]) that if $S$ is discrete and left reversible, then $S$ has the following fixed point property:

(G): Whenever $S$ acts on a weakly compact convex subset $K$ of a separated locally compact convex space $(E, Q)$ and the action 
is weakly separately continuous and $Q$-non-expansive, then $K$ contains a common fixed point for $S$.

Since the fixed point property $(\mathrm{G})$ implies that $W A P(S)$ has LIM, it follows that if $S$ is discrete and left reversible, then $W A P(S)$ has a LIM. This improved an earlier result of Ryll-Nardzewski who proved, using his fixed point theorem for affine maps on weakly compact convex subsets of a Banach space, the existence of LIM on $W A P(S)$ when $\mathrm{S}$ is a group (see [24]).

It is known that if $S$ is discrete and left amenable, then $S$ is left reversible. However a general semitopological semigroup $S$ needs not be left reversible even when $C_{b}(S)$ has a LIM unless $S$ is normal (see [27]).

The following implication diagram summarizes the known relations mentioned above for a discrete semigroup $S$.

$S$ left amenable

$\Downarrow \not{\not}$

$S$ left reversible

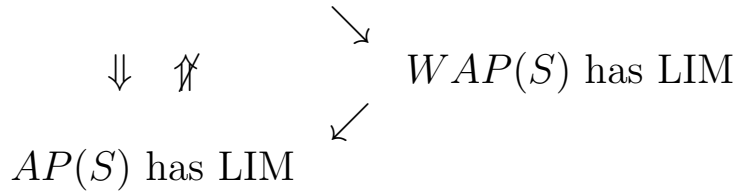

The implication " $S$ is left reversible $\Rightarrow A P(S)$ has a LIM" for any semitopological semigroup was established in [36]. During the 1984 Richmond, Virginia conference on analysis on semigroups, T. Mitchell gave two examples to show that, for a discrete semigroup $S$, " $A P(S)$ has LIM" $\nRightarrow$ "S is left reversible" (see [39]).

\section{Semigroups OF NON-EXPANSIVE MAPPINGS}

An action of a semitopological semigroup $S$ on a Hausdorff space $X$ is called quasi-equicontinuous if $\bar{S}^{p}$, the closure of $S$ in the product space $X^{X}$, consists of only continuous mappings. Obviously, an equicontinuous action on a closed subset of a topological vector space is always quasi-equicontinuous (simply because if a net of equicontinuous functions converges pointwise to a function, then the limit function is also continuous). But a quasi-equicontinuous action on a convex compact subset of a topological vector space may not be equicontinuous ( $[55$, Example 4.14]). The following was proved in [55].

Theorem 2.1. Let $S$ be a separable semitopological semigroup. Then $W A P(S)$ has a LIM if and only if $S$ has the fixed point property (F) stated as follows. 
(F): Whenever $S$ acts on a weakly compact convex subset $K$ of a separated locally convex space $(E, Q)$ and the action is weakly separately continuous, weakly quasi-equicontinuous and $Q$-nonexpansive, then $K$ contains a common fixed point for $S$.

Consider the following fixed point property for a semitopological semigroup $S$ :

(E): Whenever $S$ acts on a weakly compact convex subset $K$ of a separated locally convex space $(E, Q)$ as $Q$-nonexpansive selfmappings and, if in addition, the action is separately continuous and equicontinuous when $K$ is equipped with the weak topology of (E.Q), then $K$ contains a common fixed point for $S$.

Clearly we have

$$
(\mathrm{G}) \Rightarrow(\mathrm{F}) \Rightarrow(\mathrm{E}) \Rightarrow(\mathrm{D})
$$

Open Problem 1. Can any of the above implications be reversed?

Related to multiplicative means, the following result was obtained in 55 .

Theorem 2.2. Let $S$ be separable and $n$ be a positive integer. Then $W A P(S)$ has a LIM of the form $\frac{1}{n} \sum_{i=1}^{n} \phi_{i}$, where each $\phi_{i}$ is a multiplicative mean on $W A P(S)$, if and only if

$\left(P_{n}\right)$ : Whenever $S$ is a separately continuous and quasi-equicontinuous action on a compact Hausdorff space $X$, then there exists a nonempty finite subset $F \subseteq X,|F| \leq n,|F|$ divides $n$ such that $s F=F$ for all $s \in S$.

Let $(E, Q)$ be a separated locally convex space. A subset $K$ of $E$ is said to have $Q$-normal structure if, for each $Q$-bounded subset $H$ of $K$ that contains more than one point, there is $x_{0} \in c o H$ and $p \in Q$ such that $\sup \left\{p\left(x-x_{0}\right): x \in H\right\}<\sup \{p(x-y): x, y \in H\}$. Here by $Q$-boundedness of $H$ we mean for each $p \in Q$ there is $d>0$ such that $p(x) \leq d$ for all $x \in H$. Any $Q$-compact subset has $Q$-normal structure. In a uniformly convex space (eg. any $L^{p}, p>1$, space) a bounded convex set always has normal structure.

For $A P(S)$ to have a LIM the following two results were proved in [55].

Theorem 2.3. Let $S$ be a semitopological semigroup. Then $A P(S)$ has $L I M$ if and only if $S$ has the following fixed point property.

$\left(\mathbf{E}^{\prime}\right)$ : Whenever $S$ acts on a weakly compact convex subset $K$ of a separated locally convex space $(E, Q)$ as $Q$-nonexpansive mappings, if $K$ has $Q$-normal structure and the $S$-action is separately continuous and equicontinuous when $K$ is equipped with 
the weak topology of $(E, Q)$, then $K$ contains a common fixed point for $S$.

In particular, fixed point properties $(D)$ and $\left(E^{\prime}\right)$ are equivalent.

Theorem 2.4. Let $S$ be a separable semitopological semigroup. Then $A P(S)$ has a LIM if and only if the fixed point property (E) holds.

We wonder whether one can remove the separability condition on $S$ in Theorems 2.1, 2.2 and 2.4. When $n=1$ we can answer this question for Theorem 2.2 affirmatively (see [56, Theorem 5.3]).

Theorem 2.5. $W A P(S)$ has a multiplicative LIM if and only if whenever $S$ is a separately continuous and quasi-equicontinuous action on a compact Hausdorff space $X$, then $X$ has a common fixed point for $S$.

The bicyclic semigroup is the semigroup generated by a unit $e$ and two more elements $p$ and $q$ subject to the relation $p q=e$. We denote it by $S_{1}=\langle e, p, q \mid p q=e\rangle$. The semigroup generated by a unit $e$ and three more elements $a, b$ and $c$ subject to the relations $a b=a c=e$ is denoted by $S_{2}=\langle e, a, b, c \mid a b=e, a c=e\rangle$; and the semigroup generated by a unit $e$ and four more elements $a, b, c, d$ subject to the relations $a c=b d=e$ is denoted by $S_{1,1}=\langle e, a, b, c, d| a c=e, b d=$ $e\rangle$. We call $S_{2}$ and $S_{1,1}$ partially bicyclic semigroups. Duncan and Namioka showed in [14] that $S_{1}$ is an amenable semigroup by revealing the maximal group homomorphic image of $S_{1}$. The authors gave a direct proof to the result in [55] by constructing a left and a right invariant mean on $\ell^{\infty}\left(S_{1}\right)$. The partially bicyclic semigroups $S_{2}$ and $S_{1,1}$ were also investigated in [55]. The results can be summarized as follows.

Theorem 2.6. Regarding the semigroups $S_{1}, S_{2}$ and $S_{1,1}$, the following properties hold.

(1) The bicyclic semigroup $S_{1}$ is amenable;

(2) The partially bicyclic semigroup $S_{1,1}$ is neither left nor right amenable, while the partially bicyclic semigroup $S_{2}$ is right amenable but not left amenable;

(3) Both $A P\left(S_{1,1}\right)$ and $A P\left(S_{2}\right)$ have an invariant mean;

(4) $W A P\left(S_{2}\right)$ has a LIM, while WAP $\left(S_{1,1}\right)$ has no LIM.

We note that both $S_{2}$ and $S_{1,1}$ are not left reversible. The above theorem shows that there are semigroups $S$ such that $A P(S)$ has a LIM but $W A P(S)$ has no LIM, and that there are semigroups $S$ such that $W A P(S)$ has a LIM but $S$ is not left reversible. So the theorem resolved problem 27 raised in [39] and problem 1 raised in [37. As 
a consequence, for a discrete semigroup $S$, the diagram (1.1) may be completed to:

left amenable $\underset{\nLeftarrow}{\Rightarrow}$ left reversible $\underset{\nLeftarrow}{\Rightarrow} W A P(S)$ has LIM $\underset{\nLeftarrow}{\Rightarrow} A P(S)$ has LIM

Consider fixed point properties $\left(\mathrm{F}^{*}\right)$ and $\left(\mathrm{G}^{*}\right)$, which are, respectively, the fixed point properties $(\mathrm{F})$ and $(\mathrm{G})$ with the words "separately continuous" replaced by "jointly continuous". Clearly, (G*) implies $\left(\mathrm{F}^{*}\right)$. The next two results were obtained in [55].

Theorem 2.7. Let $S$ be a separable semitopological semigroup. Then fixed point property $\left(F^{*}\right)$ holds if and only if the space $W A P(S) \cap$ $\operatorname{LUC}(S)$ has a LIM.

Theorem 2.8. Let $S$ be a left reversible and metrizable semitopological semigroup. Then $S$ has the fixed point property $\left(G^{*}\right)$. In particular, $W A P(S) \cap L U C(S)$ has a LIM.

The following diagram summarizes the relations among the fixed point properties discussed in [55].

$$
\begin{aligned}
& \begin{array}{l}
S \text { is metrizable } \\
\text { \& left reversible }
\end{array}\left(G^{*}\right) \Rightarrow \quad\left(F^{*}\right) \quad \stackrel{\text { s }}{\Leftrightarrow} W A P(S) \cap L U C(S) \text { has LIM }
\end{aligned}
$$

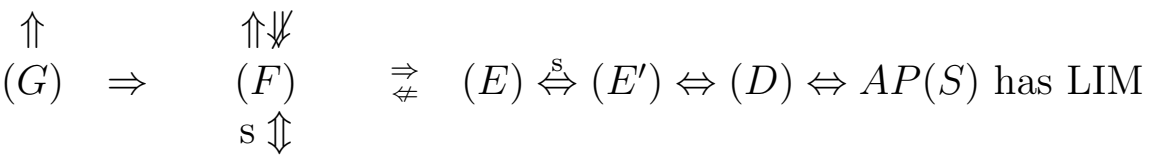

$$
\begin{aligned}
& W A P(S) \\
& \text { has LIM }
\end{aligned}
$$

where "s" means the implication is under the condition that the semigroup is separable.

Let $S$ be a semitopological semigroup. The set $\operatorname{MM}(S)$ of all multiplicative means on $C_{b}(S)$ is a total subset of $C_{b}(S)^{*}$. It generates a locally convex topology $\tau_{M M}=\sigma\left(C_{b}(S), \mathrm{MM}(S)\right)$ on $C_{b}(S)$. A function $f \in C_{b}(S)$ is called left multiplicatively continuous if the mapping $s \mapsto \ell_{s} f$ from $S$ into $\left(C_{b}(S), \tau_{M M}\right)$ is continuous [66]. Let $L M C(S)$ be the subspace of $C_{b}(S)$ consisting of all left multiplicatively continuous functions on $S$. Then $L M C(S)$ is a left invariant closed subalgebra of $C_{b}(S)$ containing the constant function. The following result is due to T. Mitchell [66]. An alternative proof was given in [56].

Theorem 2.9. Let $S$ be a semitopological semigroup. Then

(1) $L U C(S)$ has a multiplicative left invariant mean if and only if $\left(F_{j}\right)$ : every jointly continuous representation of $S$ on a nonempty compact Hausdorff space has a common fixed point for $S$; 
(2) $L M C(S)$ has a multiplicative left invariant mean if and only if $\left(F_{s}\right)$ : every separately continuous representation of $S$ on a nonempty compact Hausdorff space has a common fixed point for $S$.

Recall further that a function $f \in C_{b}(S)$ is a weakly left uniformly continuous if the mapping $s \mapsto \ell_{s} f$ from $S$ into $\left(C_{b}(S)\right.$, wk) is continuous [66]. The set of all weakly left uniformly continuous functions on $S$ is a left invariant closed subspace of $C_{b}(S)$, denoted by $W L U C(S)$. Denote by $\mathrm{M}(S)$ the set of all means on $C_{b}(S)$ and let $\tau_{M}=\sigma\left(C_{b}(S), \mathrm{M}(S)\right)$. Since $C_{b}(S)^{*}$ is linearly spanned by $\mathrm{M}(S)$, we have that $f \in W L U C(S)$ if and only if the mapping $s \mapsto \ell_{s} f$ from $S$ into $\left(C_{b}(S), \tau_{M}\right)$ is continuous. The following theorem is also due to $\mathrm{T}$. Mitchell [66]. An alternative proof was given in [56].

Theorem 2.10. Let $S$ be a semitopological semigroup. Then

(1) $L U C(S)$ has a left invariant mean if and only if

$\left(F_{j a}\right)$ : every jointly continuous affine representation of $S$ on a nonempty convex compact subset of a separated locally convex topological vector space has a common fixed point for $S$;

(2) $W L U C(S)$ has a left invariant mean if and only if

$\left(F_{\text {sa }}\right)$ : every separately continuous affine representation of $S$ on a compact convex subset of a separated locally convex topological vector space has a common fixed point for $S$.

Remark 2.11. We note that Theorem 2.10.(1) is no longer true if one replaces "affine" with "convex". Here by a convex map (or a convex function) we mean a map (resp. a function) defined on a convex set $K$ that maps each convex subset of $K$ onto a convex set. For example any continuous function on an interval is a convex function. A counter example is as follows: Let $f$ and $g$ be two continuous functions on the unit interval $[0,1]$ such that $f$ commutes with $g$ under composite product but they do not have a common fixed point in $[0,1]$. We consider the free discrete commutative semigroup $S C_{2}$ on two generators $s_{1}$ and $s_{2}$. Then $S C_{2}$ is amenable. Define on $[0,1]$ by $T_{s_{1}}(x)=f(x)$ and $T_{s_{2}}(x)=g(x)(x \in[0,1])$. They generate a continuous convex representation of $S C_{2}$ on $[0,1]$. This representation does not have a common fixed point in $[0,1]$.

\section{FIXED POINT PROPERTIES FOR L-EMBEDDED SETS}

A Banach space $E$ is L-embedded if the image of $E$ under the canonical embedding into $E^{* *}$, the bidual space of $E$, is an $\ell_{1}$ summand in 
$E^{* *}$. The Banach space $E$ is M-embedded if $E$ is an M-ideal in its bidual $E^{* *}$, that is, if $E^{\perp}=\left\{\phi \in E^{* * *}: \phi(x)=0\right.$ for all $\left.x \in E\right\}$ is an $\ell_{1}$-summand in $E^{* * *}([26])$. In fact, $E$ is M-embedded if and only if $E^{* * *}=E^{*} \oplus_{1} E^{\perp}([26$, Remark 1.13]). The class of L-embedded Banach spaces includes all $L^{1}(\Sigma, \mu)$ (the space of all absolutely integrable functions on a measure space $(\Sigma, \mu)$ ), preduals of von Neumann algebras and the Hardy space $H_{1}$. A typical example of an M-embedded Banach space is $c_{0}(D)$ for a discrete space $D$ (see [56, page 2953]). It is also well-known that $K(H)$, the Banach space of all compact operators on a Hilbert space $H$, is M-embedded. More classical M-embedded Banach spaces may be seen in [26, Example 1.4]. The dual space of an M-embedded Banach space is L-embedded.

The authors introduced the notion of L-embedded subsets of Banach spaces in [56].

Definition 3.1. Let $C$ be a nonempty subset of a Banach space $E$. Denote by $\bar{C}^{\text {wk* }}$ the closure of $C$ in $E^{* *}$ in the weak topology of $E^{* *}$. We call $C L$-embedded if there is a subspace $E_{s}$ of $E^{* *}$ such that $E+$ $E_{s}=E \oplus_{1} E_{s}$ in $E^{* *}$ and $\bar{C}^{\mathrm{wk}^{*}} \subset C \oplus_{1} E_{s}$, that is, for each $u \in \bar{C}^{\mathrm{wk}^{*}}$ there are $c \in C$ and $\xi \in E_{s}$ such that $u=c+\xi$ and $\|u\|=\|c\|+\|\xi\|$.

Trivially, every L-embedded Banach space is L-embedded as a subset of itself. From the definition, every L-embedded set $C$ in a Banach space $E$ is necessarily weakly closed.

Every weakly compact subset $C$ of a Banach space is L-embedded since $\bar{C}^{\text {wk* }^{*}}=C$ in the case. A Banach space $E$ is L-embedded if and only its unit ball is L-embedded subset of $E$. But a closed convex subset of an L-embedded Banach space may not be L-embedded. For example, the set of all means on $\ell^{\infty}$ is a convex closed (and even weak* compact) subset of $\left(\ell^{\infty}\right)^{*}$, but it is not L-embedded although $\left(\ell^{\infty}\right)^{*}$ (as the dual space of a von Neumann algebra) is L-embedded. For a $\sigma$-finite measure space $(\Sigma, \mu)$, it was shown in [9, Theorem 1.1] that a closed convex bounded set in $L^{1}(\Sigma, \mu)$ is L-embedded if and only if it is closed with respect to the local measure topology. A generalization of this result in the operator algebra setting was given in [12, Theorem 3.5].

The following result was shown in [56].

Theorem 3.2. Let $S$ be a left reversible discrete semigroup. Then $S$ has the following fixed point property.

$\left(F_{L}\right):$ Whenever $\mathcal{S}=\left\{T_{s}: s \in S\right\}$ is a representation of $S$ as norm nonexpansive self mappings on a nonempty L-embedded convex subset $C$ of a Banach space $E$ with each $T_{s}$ being weakly continuous on every weakly compact $S$-invariant convex subset 
of $C$, if $C$ contains a nonempty bounded subset $B$ such that $T_{s}(B)=B$ for all $s \in S$, then $C$ has a common fixed point for $S$.

Recall that a semitopological semigroup $S$ is strongly left reversible if there is a family of countable subsemigroups $\left\{S_{\alpha}: \alpha \in I\right\}$ such that

(1) $S=\cup_{\alpha \in I} S_{\alpha}$,

(2) $\overline{a S_{\alpha}} \cap \overline{b S_{\alpha}} \neq \emptyset$ for each $\alpha \in I$ and $a, b \in S_{\alpha}$,

(3) for each pair $\alpha_{1}, \alpha_{2} \in I$, there is $\alpha_{3} \in I$ such that $S_{\alpha_{1}} \cup S_{\alpha_{2}} \subset$ $S_{\alpha_{3}}$.

A metrizable left reversible semitopological semigroup is always strongly left reversible [55, Lemma 5.2]. Using this fact and the same proof as for Theorem 3.2 one can derive the following theorem.

Theorem 3.3. Let $S$ be a metrizable left reversible semitopological semigroup. Let $\mathcal{S}=\left\{T_{s}: s \in S\right\}$ be a norm nonexpansive representation of $S$ on a nonempty L-embedded convex subset $C$ of a Banach space $E$ such that $C$ contains a nonempty bounded subset $B$ with $T_{s}(B)=B$ for all $s \in S$. If for every weakly compact $S$-invariant convex subset $M$ of $C$ the mapping $(s, x) \mapsto T_{s}(x)$ is jointly continuous from $S \times M$ into $M$ when $M$ is endowed with the weak topology of $E$, then $C$ has a common fixed point for $S$.

Again, the same method combined with [61, Theorem 3] leads to the following theorem.

Theorem 3.4. Let $S$ be a left reversible semitopological semigroup and let $\mathcal{S}=\left\{T_{s}: s \in S\right\}$ be a representation of $S$ as norm nonexpansive and separately continuous self mappings on a nonempty L-embedded convex subset $C$ of a Banach space $E$. Suppose that $C$ has normal structure and contains a nonempty bounded subset $B$ such that $T_{s}(B)=B$ for all $s \in S$. Then $C$ has a common fixed point for $S$.

For affine nonexpansive mappings on a closed convex L-embedded set of a Banach space the following was obtained in [56].

Theorem 3.5. Let $C$ be a nonempty closed convex set in a Banach space and $S$ be a semitopological semigroup such that $W A P(S)$ has a LIM. Suppose that $\mathcal{S}=\left\{T_{s}: s \in S\right\}$ is a representation of $S$ as separately continuous nonexpansive affine mappings on $C$. If $C$ is $L$ embedded and there is a bounded set $B \subset C$ such that $T_{s}(B)=B$ for all $s \in S$, then $C$ has a common fixed point for $S$.

If $S$ is a locally compact group then $W A P(S)$ always has a LIM and, for each $x \in C, B=S x$ always satisfies $T_{s}(B)=B$ for all $s \in S$. 
So, if there is $x \in C$ such that $S x$ is bounded, then every separately continuous nonexpansive affine representation of $S$ on a nonempty Lembedded convex set of a Banach space has a common fixed point for $S$. Thus, Theorem 3.5] extends [3, Theorem A] of Bader, Gelander and Monod, which was used to give a short proof to resolve the derivation problem. This long-standing problem was first solved by V. Losert in 63 .

For a discrete semigroup $S$ it is well-known that the left reversibility of $S$ implies that $W A P(S)$ has a left invariant mean and the converse is not true due to Theorem 2.6. For general semitopological semigroups $S$, the relation between the left reversibility of $S$ and the existence of a left invariant mean for $W A P(S)$ is still unknown.

We recall that if $S$ acts on a Hausdorff space $X$, the action is quasiequicontinuous if $\bar{S}^{p}$, the closure of $S$ in the product space $X^{X}$, consists entirely of continuous mappings. Let $C$ be a closed convex subset of a Banach space $E$ and let $S$ act on $C$ as self mappings. We say that the action is hereditary weakly quasi-equicontinuous in $C$ if for each weakly compact $S$-invariant convex subset $K$ of $C$ the $S$-action on $(K$, wk) is quasi-equicontinuous, where $(K$, wk) is $K$ with the weak topology of $E$. We note that, if the $S$-action on $C$ is weakly equicontinuous, then it is hereditary quasi-equicontinuous in $C$. By [55, Lemma 3.1], if $C$ is weakly compact and the $S$-action on $C$ is weakly quasi-equicontinuous, then the $S$-action is hereditary weakly quasi-equicontinuous in $C$. The following was shown in [56].

Theorem 3.6. Let $S$ be a separable semitopological semigroup. Suppose that $W A P(S)$ has a left invariant mean. Then $S$ has the following fixed point property.

$\left(F_{L}^{\prime}\right)$ : If $S$ acts on a nonempty convex L-embedded subset $C$ of a Banach space as norm nonexpansive and hereditary weakly quasi-equicontinuous mappings for which the mapping $s \mapsto T_{s}(x)$ $(s \in S)$ is weakly continuous whenever $x$ belongs to any weakly compact $S$-invariant convex subset of $C$ and if $C$ contains a nonempty bounded subset $B$ such that $T_{s}(B)=B(s \in S)$, then there is a common fixed point for $S$ in $C$.

The above theorem was used in [56] to provide the following example.

Example. Let $G$ be a non-amenable group and let $C$ be the set of all means on $\ell^{\infty}(G)$. Then $C$ is not L-embedded although it is a weak* compact convex subset of $\ell^{\infty}(G)^{*}$ which, as the dual space of the von Neumman algebra $\ell^{\infty}(G)$, is indeed an L-embedded Banach space. As a consequence, the set of all means on $\ell^{\infty}$ is not L-embedded. 
For a semitopological semigroup $S$, simply examining the representation of $S$ on the weak* compact convex subset of all means on $L U C(S)$ defined by the dual of left translations on $L U C(S)$, one sees that if the following fixed point property holds then $L U C(S)$ has a left invariant mean.

$\left(F_{*}\right)$ : Whenever $\mathcal{S}=\left\{T_{s}: s \in S\right\}$ is a representation of $S$ as norm non-expansive mappings on a non-empty weak* compact convex set $C$ of the dual space of a Banach space $E$ and the mapping $(s, x) \mapsto T_{s}(x)$ from $S \times C$ to $C$ is jointly continuous, where $C$ is equipped with the weak ${ }^{*}$ topology of $E^{*}$, then there is a common fixed point for $S$ in $C$.

Whether the converse is true is an open problem raised in [40]. For a discrete semigroup acting on a subset of the dual of an M-embedded Banach space, the following was given in [56].

Theorem 3.7. Let $S$ be a discrete left reversible semigroup. Then $S$ has the following fixed point property.

$\left(F_{* M}\right):$ Whenever $\mathcal{S}=\left\{T_{s}: s \in S\right\}$ is a representation of $S$ as weak $^{*}$ continuous, norm nonexpansive mappings on a nonempty weak $^{*}$ compact convex set $C$ of the dual space $E^{*}$ of an $M$ embedded Banach space $E, C$ contains a common fixed point for $S$.

For non-discrete $S$ we have:

Theorem 3.8. Let $S$ be a left reversible semitopological semigroup. Then $S$ has the following fixed point property.

$\left(F_{* M j}\right):$ If $\mathcal{S}=\left\{T_{s}: s \in S\right\}$ is a norm nonexpansive representation of $S$ on a nonempty weak* compact convex set $C$ of the dual space $E^{*}$ of an $M$-embedded Banach space $E$ and the mapping $(s, x) \mapsto T_{s}(x)$ from $S \times C$ into $C$ is jointly continuous when $C$ is endowed with the weak* topology of $E^{*}$, then $C$ contains a common fixed point for $S$.

Remark 3.9. Since the Banach space $K(H)$ of all compact operators on a Hilbert space $H$ is M-embedded, it follows that both Theorems 3.7 and 3.8 apply to $K(H)^{*}=J(H)$, the space of trace operators on $H$.

\section{FIXED POINT PROPERTIES FOR F-ALGEBRAS}

In [17] (see also [18, 19, 43, 50, 51]), Ky Fan established the following remarkable "Invariant Subspace Theorem" for left amenable semigroups: 
Theorem 4.1. Let $S$ be a left amenable semigroup, and let $\mathcal{S}=\left\{T_{s}\right.$ : $s \in S\}$ be a representation of $S$ as continuous linear operators on a separated locally convex space $E$. Then the following property holds:

(KF): If $X$ is a subset of $E$ (containing an n-dimensional subspace) such that $T_{s}(L)$ is an $n$-dimensional subspace contained in $X$ whenever $L$ is one and $s \in S$, and there exists a closed $\mathcal{S}$ invariant subspace $H$ in $E$ of codimension $n$ with the property that $(x+H) \cap X$ is compact convex for each $x \in E$, then there exists an $n$-dimensional subspace $L_{0}$ contained in $X$ such that $T_{s}\left(L_{0}\right)=L_{0}$ for all $s \in S$.

The origin of Ky Fan's Theorem lies in the earlier investigations of Pontrjagin, Iovihdov, Krein and Naimark concerning invariant subspaces for Lorentz transformations on a Hilbert space [32, 33, 35, 69, 70, 72. In physics, a Lorentz transformation is an invertible linear mapping on $\mathbb{R}^{4}$ that describes how a measurement of space and time observed in a frame of reference is converted into another frame of reference. From special relativity Lorentz transformations may be characterized as invertible linear mappings that preserve the quadratic form

$$
J(\vec{x})=x^{2}+y^{2}+z^{2}-c^{2} t^{2}, \quad \vec{x}=(x, y, z, t) \in \mathbb{R}^{4}
$$

where the constant $c$ is the speed of light. Quantity $J$ represents the space time interval. It is a well known fact that for any Lorentz transformation $T$ there is a three dimensional subspace $V$ of $\mathbb{R}^{4}$ which is $T$-invariant and positive (in the sense that $T(\vec{x})=\vec{x}$ and $J(\vec{x}) \geq 0$ for all $\vec{x} \in V)$.

L. S. Pontrjagin [72], I. S. Iovihdov [32], M. G. Krein [35, 33] and M. A. Naimark [69, 70] investigated infinite-dimension versions of the above invariant subspace property, and Naimark finally established the following theorem in 1963 [69].

Theorem 4.2. Let $n>0$ be an integer. Consider the quadratic form on $\ell^{2}$ given by

$$
J_{n}(x)=\sum_{i=1}^{n}\left|x_{i}\right|^{2}-\sum_{i=n+1}^{\infty}\left|x_{i}\right|^{2}, \quad x=\left(x_{1}, x_{2}, \cdots\right) \in \ell^{2} .
$$

Suppose that $G$ is a commutative group of continuous, invertible, $J_{n^{-}}$ preserving, linear transformations on $\ell^{2}$. Then there is a $G$-invariant $n$-dimensional subspace $V$ of $\ell^{2}$ which is positive (in the sense that $J_{n}(x) \geq 0$ for all $\left.x \in V\right)$.

To understand the conditions on the subset $X$ in Ky Fan's property (KF) we note that $X=\left\{x \in \ell^{2}: J_{n}(x) \geq 0\right\}$ in the setting of 
the above theorem satisfies indeed the requirement in $(\mathrm{KF})$. However, there is no mention of an invariant subspace $H$ in Naimark's result. Ky Fan's Theorem removes the commutative group and Hilbert space restrictions of Naimark's Theorem, replacing them by amenability and the conditions involving $H$.

Let $A$ be a Banach algebra and let $X$ be a Banach left, right or two-sided $A$-module. Then $X^{*}$ is respectively a Banach right, left or two-sided $A$-module with the corresponding module action(s) defined naturally by

$$
\langle x, f \cdot a\rangle=\langle a \cdot x, f\rangle,\langle x, a \cdot f\rangle=\langle x \cdot a, f\rangle \quad\left(a \in A, f \in X^{*}, x \in X\right) .
$$

Let $X$ be a Banach $A$-bimodule. A linear mapping $D: A \rightarrow X$ is called a derivation if it satisfies

$$
D(a b)=a \cdot D(b)+D(a) \cdot b \quad(a, b \in X) .
$$

Derivations in the form $D(a)=a \cdot x_{0}-x_{0} \cdot a(a \in A)$ for some fixed $x_{0} \in X$ are called inner derivations.

A Banach algebra $A$ is an F-algebra [42] (also known as Lau algebras [71]) if it is the (unique) predual of a $W^{*}$-algebra $\mathfrak{M}$ and the identity $\mathfrak{e}$ of $\mathfrak{M}$ is a multiplicative linear functional on $A$. Since $A^{* *}=\mathfrak{M}^{*}$, we denote by $P_{1}\left(A^{* *}\right)$, the set of all normalized positive linear functionals on $\mathfrak{M}$, that is

$$
P_{1}\left(A^{* *}\right)=\left\{m \in A^{* *}: m \geq 0, m(\mathfrak{e})=1\right\} .
$$

In this case $P_{1}\left(A^{* *}\right)$ is a semigroup with the (two) Arens multiplications.

Examples of F-algebras include the predual algebras of a Hopf von Neumann algebra (in particular, quantum group algebras), the group algebra $L^{1}(G)$ of a locally compact group $G$, the Fourier algebra $A(G)$ and the Fourier-Stieltjes algebra $B(G)$ of a topological group $G$ (see [10, 42, 47]). They also include the measure algebra $M(S)$ of a locally compact semigroup $S$. Moreover, the hypergroup algebra $L^{1}(H)$ and the measure algebra $M(H)$ of a locally compact hypergroup $H$ with a left Haar measure are F-algebras. In this case, it was shown in [82, Theorem 5.2.2] (see also [83, Remark 5.3]) that $\left(L^{1}(H)\right)^{*}=L^{\infty}(H)$ is not a Hopf von Neumann algebra unless $H$ is a locally compact group.

Let $G$ be a locally compact group with a fixed left Haar measure $\lambda$. Then the group algebra $L^{1}(G)$ is the Banach space of $\lambda$-integrable functions with product

$$
f * g(x)=\int_{G} f(y) g\left(y^{-1} x\right) d \lambda(y) \quad(x \in G) .
$$


If $S$ is a locally compact semigroup, then the measure algebra $M(S)$ is the space of regular Borel measures on $S$ with the total variation norm and the convolution product defined by

$$
\langle\mu * \nu, f\rangle=\iint_{G \times G} f(x y) d \nu(y) d \mu(x) \quad\left(f \in C_{0}(S)\right),
$$

where $C_{0}(S)$ denotes the space of continuous functions vanishing at $\infty$. If $S$ is discrete, then $M(S)=\ell^{1}(S)$.

In his seminal paper [16] P. Eymard (see also [30]) associated to a locally compact group $G$ two important commutative Banach algebras. These are the Fourier algebra $A(G)$ and the Fourier-Stieltjes algebra $B(G)$. The latter is indeed the linear span of the set of all continuous positive definite complex-valued functions on $G$. This is also the space of the coefficient functions of the unitary representations of the group $G$. More precisely, given $u \in B(G)$ there exists a unitary representation $\pi$ of $G$ and two vectors $\xi$ and $\eta$ in the representation Hilbert space $H(\pi)$ of $\pi$ such that

$$
u(x)=\langle\pi(x) \xi, \eta\rangle \quad(x \in G) .
$$

Equipped with the norm $\|u\|=\inf _{\xi, \eta}\|\xi\|\|\eta\|$ and the pointwise multiplication $B(G)$ is a commutative Banach algebra, where the infimum is taking on all $\xi$ and $\eta$ satisfying the preceding equality. As a Banach algebra $B(G)$ is also the dual space of the group $\mathrm{C}^{*}$-algebra $C^{*}(G)$. The Fourier algebra $A(G)$ is the closed ideal of $B(G)$ generated by the elements of $B(G)$ with compact supports. The algebra $A(G)$ can also be defined as the set of coordinate functions of the left regular representations of $G$ on $L^{2}(G)$. When $G$ is abelian, via Fourier transform we have

$$
A(G)=L^{1}(\hat{G}), \quad B(G)=M(\hat{G}) \quad \text { and } C^{*}(G)=C_{0}(\hat{G}),
$$

where $\hat{G}$ is the dual group of $G$.

We call a semitopological semigroup $S$ extremely left amenable if there is a left invariant mean $m$ on $\operatorname{LUC}(S)$ which is multiplicative, that is it satisfies further

$$
m(f g)=m(f) m(g) \quad(f, g \in L U C(S)) .
$$

Let $A$ be an F-algebra. Elements of $P_{1}\left(A^{* *}\right)$ are called means on $A^{*}=\mathfrak{M}$. It is well-known that

$$
P_{1}(A)=P_{1}\left(A^{* *}\right) \cap A
$$

is a topological semigroup with the product and topology carried from $A$ (this may be regarded as a consequence of [75, Proposition 1.5.2]), and $P_{1}(A)$ spans $A$ (see [75, Theorem 1.14.3]). A mean $m \in P_{1}\left(A^{* *}\right)$ 
on $A^{*}$ is called a topological left invariant mean, abbreviated as TLIM, if $a \cdot m=m$ for all $a \in P_{1}(A)$, in other words $m \in P_{1}\left(A^{* *}\right)$ is a TLIM if $m(x \cdot a)=m(x)$ for all $a \in P_{1}(A)$ and $x \in A^{*}$.

An F-algebra $A$ is called left amenable if, for each Banach $A$-bimodule $X$ with the left module action specified by $a \cdot x=\langle a, \mathfrak{e}\rangle x(a \in A, x \in X)$, every continuous derivation from $A$ into $X^{*}$ is inner. The following was shown in [42] (see Theorems 4.1 and 4.6 there).

Proposition 4.3. Let $A$ be an F-algebra. Then the following are equivalent.

(1) There is a TLIM for $A^{*}$.

(2) The algebra $A$ is left amenable.

(3) There exists a net $\left(m_{\alpha}\right) \subset P_{1}(A)$ such that am $m_{\alpha}-m_{\alpha} \rightarrow 0$ in norm topology for each $a \in P_{1}(A)$.

We note here that, being F-algebras, the group algebra $L^{1}(G)$, the measure algebra $M(G)$ of a locally compact group $G$ are left amenable if and only if $G$ is an amenable group; while the Fourier algebra $A(G)$ and the Fourier-Stieltjes algebra $B(G)$ are always left amenable [42]. The hypergroup algebra $L^{1}(H)$ of a locally compact hypergroup $H$ with a left Haar measure is left amenable if and only if $H$ is an amenable hypergroup [76]. Also the left amenability of the predual algebra of a Hopf von Neumann algebra, as an F-algebra, coincides with that studied in [74, 81] (see also [4] and references therein).

A metric semigroup is a semitopological semigroup whose topology is generated by a metric $d$. We consider the following fixed point property for a metric semigroup $S$.

$\left(F_{U}\right):$ If $\mathcal{S}=\left\{T_{s}: s \in S\right\}$ is a separately continuous representation of $S$ on a compact subset $K$ of a locally convex space $(E, Q)$ and if the mapping $s \mapsto T_{s}(y)$ from $S$ into $K$ is uniformly continuous for each $y \in K$, then $K$ has a common fixed point for $S$.

Note that the mapping $s \mapsto T_{s}(y)$ is uniformly continuous if for each $\tau \in Q$ and each $\epsilon>0$ there is $\delta>0$ such that

$$
\tau\left(T_{s}(y)-T_{t}(y)\right) \leq \epsilon
$$

whenever $d(s, t) \leq \delta$. For example, suppose that $S$ is a subset of a locally convex space $L$ that acts on $E$ such that $(a, y) \mapsto a y: L \times E \rightarrow E$ is separately continuous; if $a \mapsto a y$ is linear in $a \in L$ for each $y \in E$, then the induced action of $S$ on $E,(s, y) \mapsto s y(s \in S)$, is uniformly continuous in $s$ for each $y \in E$. 
Let $A$ be an F-algebra. As we have known, $P_{1}(A)$ is indeed a metric topological semigroup with the product and topology inherited from $A$. The following was proved in [57].

Proposition 4.4. The F-algebra $A$ is left amenable if and only if the metric semigroup $P_{1}(A)$ has the fixed point property $\left(F_{U}\right)$.

A semitopological semigroup $S$ is extremely left amenable if $L U C(S)$ has a multiplicative left invariant mean. Granirer showed in $[22]$ that a discrete semigroup is extremely left amenable if and only if any two elements of it have a common right zero (see [56, Theorem 4.2] for a short proof). It is due to Mitchell [66 that a semitopological semigroup $S$ is extremely left amenable if and only if it has the following fixed point property.

$\left(F_{E}\right)$ : Every jointly continuous representation of $S$ on a compact Hausdorff space $C$ has a common fixed point in $C$.

For an F-algebra $A$ it is pleasing that the left amenability of $A$ is equivalent to the extreme left amenability of $P_{1}(A)$ as the authors revealed in [57].

Theorem 4.5. Let $A$ be an F-algebra. Then $A$ is left amenable if and only if $P_{1}(A)$ has the fixed point property $\left(F_{E}\right)$.

According to Theorem 4.5, we can characterize amenability of a group (resp. semigroup) in terms of the fixed point property of normalized positive functions in the group/semigroup algebra.

Corollary 4.6. Let $G$ be a locally compact group and let $S$ be a semigroup. Then

(1) The group $G$ is amenable if and only if the metric semigroup $P_{1}(G)=\left\{f \in L^{1}(G), f \geq 0,\|f\|_{1}=1\right\}$ has the fixed point property $\left(F_{E}\right)$.

(2) The semigroup $S$ is left amenable if and only if the metric semigroup $P_{1}(S)=\left\{f \in \ell^{1}(S), f \geq 0,\|f\|_{1}=1\right\}$ has the fixed point property $\left(F_{E}\right)$.

Remark 4.7. For $A=A(G)$, the Fourier algebra of a locally compact group $G$, the necessity part of Theorem 4.5 was obtained earlier in [41].

Remark 4.8. From Theorem 4.5, a locally compact group is amenable if and only if $L U C\left(S_{G}\right)$ has a multiplicative left invariant mean, where $S_{G}$ is the metric semigroup $P_{1}(G)=\left\{f \in L^{1}(G): f \geq 0,\langle f, 1\rangle=1\right\}$. As a consequence, $A P\left(S_{G}\right)$ has a left invariant mean (which is equivalent to the left reversibility of $\overline{\left(S_{G}\right)^{a}}$, the almost periodic compactification of $S_{G}$ ) if $G$ is amenable. 
Remark 4.9. A common fixed point property for affine actions of $P_{1}(A)$ with a weak topology on compact convex sets has been studied in [11] for left amenable F-algebras $A$.

Let $E$ be a separated locally convex vector space and $X$ a subset of $E$. Given an integer $n>0$ we denote by $\mathcal{L}_{n}(X)$ the collection of all $n$-dimensional subspaces of $E$ that are included in $X$. Let $S$ be a semigroup and $\mathcal{S}=\left\{T_{s}: s \in S\right\}$ a linear representation of $S$ on $E$. We say that $X$ is $n$-consistent with respect to $S$ if $\mathcal{L}_{n}(X) \neq \emptyset$ and $\mathcal{L}_{n}(X)$ is $S$-invariant, that is $T_{s}(L) \in \mathcal{L}_{n}(X)$ for all $s \in S$ whenever $L \in \mathcal{L}_{n}(X)$. We say that the representation $\mathcal{S}$ is jointly continuous on compact sets if the following is true: For each compact set $K \subset E$, if $\left(s_{\alpha}\right) \subset S$ and $\left(x_{\alpha}\right) \subset K$ are such that $s_{\alpha} \stackrel{\alpha}{\rightarrow} s \in S, x_{\alpha} \stackrel{\alpha}{\rightarrow} x \in K$ and $T_{s_{\alpha}}\left(x_{\alpha}\right) \in K$ for all $\alpha$, then $T_{s_{\alpha}}\left(x_{\alpha}\right) \stackrel{\alpha}{\rightarrow} T_{s}(x)$. Obviously, if the mapping $(s, x) \mapsto T_{s}(x)$ : $S \times E \rightarrow E$ is continuous, then $\mathcal{S}$ is jointly continuous on compact sets. The following result was proved in [57].

Theorem 4.10. Let $A$ be an F-algebra. If $A$ is left amenable then $S=P_{1}(A)$ has the following $n$-dimensional invariant subspace property for each $n>0$.

$\left(F_{n}\right)$ : Let $E$ be a separated locally convex vector space and $\mathcal{S}$ a linear representation of $S=P_{1}(A)$ on $E$ such that the mapping $s \mapsto T_{s}(x)$ is continuous for each fixed $x \in E$ and $\mathcal{S}$ is jointly continuous on compact subsets of $E$. If $X$ is a subset of $E n$ consistent with respect to $S$, and if there is a closed $S$-invariant subspace $H$ of $E$ with codimension $n$ such that $(x+H) \cap X$ is compact for each $x \in E$, then there is $L_{0} \in \mathcal{L}_{n}(X)$ such that $T_{s}\left(L_{0}\right)=L_{0}(s \in S)$.

Conversely, if $\left(F_{n}\right)$ holds for some $n>0$, then $A$ is left amenable.

As a consequence of Theorems 4.10 and 4.5 we can get the following result that settles the open problem 5 in [44].

Corollary 4.11. Let $\mathbb{G}$ be a locally compact quantum group. Then $L^{1}(\mathbb{G})$ is left amenable if and only if the topological semigroup $P_{1}\left(L^{1}(\mathbb{G})\right)$ has the fixed point property $\left(F_{E}\right)$ if and only if $P_{1}\left(L^{1}(\mathbb{G})\right)$ has the finite invariant subspace property $\left(F_{n}\right)$ for some integer (and then for all integers) $n \geq 1$.

\section{Nonlinear ACTIONS ON UNBOUNDED SETS}

In [2] Atsushiba and Takahashi introduced the concept of common attractive points for a nonexpansive representation of a semigroup $S$ on 
a set $C$ in a Hilbert space $H$. They showed that $F(S) \neq \emptyset$ for commutative $S$ if there is a common attractive point for $S$ [2, Lemma 3.1]. They showed further that for commutative semigroups $S$, if $\left\{T_{s} c, s \in S\right\}$ is bounded for some $c \in C \subset H$, then the set $A_{C}(\mathcal{S})$ of all attractive points of $\mathcal{S}$ is not empty. As a consequence, $F(S) \neq \emptyset[2$, Theorem 4.1]. We note that the assumption that $\left\{T_{s} c, s \in S\right\}$ is bounded for some $c \in C \subset H$ cannot be dropped. Indeed, by a classical result of W. Ray in [73], for every unbounded convex subset $C$ of a Hilbert space there is a nonexpansive mapping $T_{0}: C \rightarrow C$ that has no fixed point in $C$. In fact, $\left\{T_{0}^{n}(c): n \in \mathbb{N}\right\}$ is unbounded for all $c \in C$. Hence the representation $\mathcal{N}=\left\{T_{0}^{n}: n \in \mathbb{N}\right\}$ of $(\mathbb{N},+)$ does not have a common fixed point in $C$. An investigation continuing that of [2] may be seen in 779 .

Let $\mathcal{S}=\left\{T_{s}: s \in S\right\}$ be a representation of a semigroup $S$ on a convex subset $C$ of a Banach space $E$. A point $a \in E$ is an attractive point of $\mathcal{S}$ if $\left\|a-T_{s} x\right\| \leq\|a-x\|$ for all $x \in C$. The set of all attractive points of $\mathcal{S}$ for $C$ is denoted by $A_{C}(\mathcal{S})$.

Recall that a Banach space $E$ is strictly convex if $\left\|\frac{x+y}{2}\right\|<1$ whenever $x, y \in E,\|x\|=\|y\|=1$ and $x \neq y$. It is readily seen that for any distinct elements $x, y_{1}, y_{2}$ from a strictly convex space with $\left\|x-y_{1}\right\|=$ $\left\|x-y_{2}\right\|=d$ we have $\left\|x-\frac{y_{1}+y_{2}}{2}\right\|<d$. E is uniformly convex if for each $0<\varepsilon \leq 2$ there exists $\delta>0$ such that $\left\|\frac{x+y}{2}\right\|<1-\delta$ whenever $x, y \in E,\|x\|=\|y\|=1$ and $\|x-y\| \geq \varepsilon$. It is known that if $E$ is uniformly convex then it is strictly convex and reflexive. Typical examples of a uniformly convex space are $L^{p}$-spaces $(p>1)$.

Now suppose $E$ is a strictly convex and reflexive Banach space. Let $C \neq \emptyset$ be a convex subset of $E$. For any $x \in E$, it was shown in [58 that there is a unique $u \in \bar{C}$, the norm closure of $C$ such that $\|u-x\| \leq\|c-x\|$ for all $c \in C$. If $C$ is closed, then we call this $u$ the metric projection of $x$ in $C$ and denote it by $P_{C}(x)$. If $E$ is a Hilbert space then $P_{C}(x)$ may also be characterized as the unique element $u \in C$ satisfying

$$
\operatorname{Re}\langle x-u \mid u-c\rangle \geq 0 \quad c \in C .
$$

Lemma 5.1. Suppose that $E$ is a strictly convex and reflexive Banach space. Let $C \neq \emptyset$ be a closed convex subset of $E$ and $\mathcal{S}$ be a representation of a semigroup $S$ on $C$. If $a \in E$ is an attractive pint of $\mathcal{S}$, then $P_{C}(a)$ is a common fixed point for $S$ in $C$.

Proof. Let $u=P_{C}(a)$. Since $a$ is attractive,

$$
\left\|a-T_{t} u\right\| \leq\|a-u\| \leq\|a-c\|
$$

for all $c \in C(t \in S)$. Thus $T_{t} u=P_{C}(a)=u$ for all $t \in S$. 
Remark 5.2. If $E$ is a general Banach space, the proof of Lemma 5.1 still works as long as $P_{C}(a)$ is uniquely defined.

The converse of Lemma 5.1 is not true in general. Namely, even if $F(S) \neq \emptyset$, it still can happen that $A_{C}(\mathcal{S})=\emptyset$. For example, let $E=\ell^{p}(p \geq 1)$. Then $C=\left\{x \in E: x=\left(x_{i}\right)_{i=1}^{\infty}, x_{1} \geq 0\right\}$ is a closed convex set in $\ell^{p}$. Consider $T\left(\left(x_{i}\right)\right)=\left(x_{1}, x_{1}, x_{2}, x_{3}, \cdots\right)$. Then $T$ is a continuous mapping on $C$, and $T$ has fixed point $\hat{0}=(0,0,0, \cdots)$. But $\mathrm{T}$ has no attractive point for $C$. As a consequence, the representation $\mathcal{N}=\left\{T^{n}: n \in \mathbb{N}\right\}$ of $(\mathbb{N},+)$ has a common fixed point in $C$ but has no attractive points for $C$.

However, if the representation is non-expansive, then any $x \in F(S)$ is clearly an attractive point of $\mathcal{S}$. Therefore, we can conclude the following.

Proposition 5.3. Let $E$ be a reflexive, strictly convex Banach space and $C \neq \emptyset$ a closed convex subset of $E$. Suppose that $\mathcal{S}$ is a representation of a semigroup $S$ on $C$ as nonexpansive self mappings. Then $A_{C}(\mathcal{S}) \neq \emptyset$ if and only if $F(S) \neq \emptyset$. Moreover, $F(S) \subset A_{C}(\mathcal{S})$.

It was shown in [58] that $A_{C}(\mathcal{S}) \neq \emptyset$ for a nonexpansive representation $\mathcal{S}$ of $S$ on a subset $C$ of a Hilbert space if $S$ has a certain amenability property. In particular, we have the following theorem.

Theorem 5.4. Let $C \neq \emptyset$ be a subset of a Hilbert space $H$ and $\mathcal{S}$ be a representation of a semitopological semigroup $S$ on $C$ as nonexpansive self mappings. Suppose that $\left\{T_{s} c: s \in S\right\}$ is bounded for some $c \in C$. Then $A_{C}(\mathcal{S}) \neq \emptyset$ if any of the following conditions holds.

(1) $C_{b}(S)$ has a LIM and the mapping $s \mapsto T_{s} c$ is continuous from $S$ into $(C, w k)$;

(2) $S$ is left amenable and the action of $S$ on $C$ is weakly jointly continuous;

(3) $A P(S)$ has a LIM and the action of $S$ on $C$ is weakly separately continuous and weakly equicontinuous continuous;

(4) $W A P(S)$ has a LIM mean and the action of $S$ on $C$ is weakly separately continuous and weakly quasi-equicontinuous.

If, in addition, $C$ is convex and closed, then $F(S) \neq \emptyset$.

For left (right) reversible semigroups we obtained the following in [58].

Theorem 5.5. Let $S$ be a left reversible and separable semitopological semigroup, and let $\mathcal{S}=\left\{T_{s}: s \in S\right\}$ be a representation of $S$ on a weakly closed subset $C \neq \emptyset$ of a Hilbert space $H$ as norm nonexpansive and weakly jointly continuous self mappings. If there is $c \in C$ such that 
$\left\{T_{s} c: s \in S\right\}$ is bounded, then $A_{C}(\mathcal{S}) \neq \emptyset$. In particular $F(S) \neq \emptyset$ if $C$ is convex.

Let $E$ be a Banach space and $C \subset E$. We call a mapping $T: C \rightarrow C$ a generalized hybrid mapping [31] if there are numbers $\alpha, \beta \in \mathbb{R}$ such that

$$
\alpha\|T x-T y\|^{2}+(1-\alpha)\|x-T y\|^{2} \leq \beta\|T x-y\|^{2}+(1-\beta)\|x-y\|^{2} .
$$

for all $x, y \in C$.

When $(\alpha, \beta)=(1,0)$, this indeed defines a nonexpansive mapping. However, the composite of two generalized hybrid mappings is usually no longer a generalized hybrid mapping, and a generalized hybrid mapping may be discontinuous. For a semigroup action generated by this type of mappings, the following results were proved in [58].

Theorem 5.6. Let $C \neq \emptyset$ be a subset of a Hilbert space $H$ and $\mathcal{S}$ be a representation of a semitopological semigroup $S$ on $C$. Suppose that $S$ is generated by a subset $\Lambda$ and, for each $s \in \Lambda, T_{s}$ is a generalized hybrid mapping on $C$, and suppose that $\left\{T_{s} c: s \in S\right\}$ is bounded for some $c \in C$. Then $A_{C}(\mathcal{S}) \neq \emptyset$ if any of the following conditions holds.

(1) $C_{b}(S)$ has a left invariant mean and the mapping $s \mapsto T_{s} c$ is continuous from $S$ into $(C, w k)$;

(2) $S$ is left amenable and the action of $S$ on $C$ is weakly jointly continuous;

(3) $A P(S)$ has a left invariant mean and the action of $S$ on $C$ is weakly separately continuous and weakly equicontinuous continuous;

(4) $W A P(S)$ has a left invariant mean and the action of $S$ on $C$ is weakly separately continuous and weakly quasi-equicontinuous.

If, in addition, $C$ is convex and closed, then $F(S) \neq \emptyset$.

\section{SUBINVARIANT SUBMEANS}

The notion of submean was first studied by Mizoguchi and Takahashi in 68. Further investigations and applications can be seen in [53, 54].

Given a set $S$, a nonempty subset $X$ of $\ell^{\infty}(S)$ is called positively semilinear if $f, g \in X$ implies $\alpha f+\beta g \in X$ for all $\alpha, \beta \in[0, \infty)$.

Let $X$ be a positively semilinear subset of $\ell^{\infty}(S)$ containing positive constants. A function $\mu: X \rightarrow \mathbb{R}$ is called a submean on $X$ if it satisfies the following conditions.

(1) If $f, g_{1}, g_{2} \in X$ and $\alpha, \beta \in[0,1]$ such that $f \leq \alpha g_{1}+\beta g_{2}$, then

$$
\mu(f) \leq \alpha \mu\left(g_{1}\right)+\beta \mu\left(g_{2}\right),
$$

(2) For every constant $c>0, \mu(c)=c$. 
It is easily seen that a submean is always continuous when $X$ is equipped with the sup norm topology of $\ell^{\infty}(S)$. A submean $\mu$ is also increasing, i.e. $\mu(f) \geq \mu(g)$ if $f, g \in X$ and $f \geq g$. We call the submean $\mu$ strictly increasing if for each constant $c>0$ there is $\delta(c)>0$ such that

$$
\mu(f+c) \geq \mu(f)+\delta(c)
$$

for all $f \in X$.

Now suppose further that $S$ is a semigroup. A submean $\mu$ on a left invariant, positively semilinear subset $X$ of $\ell^{\infty}(S)$ containing positive constants is called left subinvariant if

$$
\mu\left(l_{s} f\right) \geq \mu(f) \quad(s \in S, f \in X) .
$$

If the equality $\mu\left(l_{s} f\right)=\mu(f)$ holds for all $s \in S$ and $f \in X$, then we call $\mu$ left invariant.

Trivially, if $X$ is a left invariant subspace of $\ell^{\infty}(S)$ containing constants, then any left invariant mean on $X$ is a strictly increasing left invariant submean on $X$. Some nonlinear examples are given as follows.

Example 1. Let $S=G$ be a group. Then

$$
\mu(f)=\sup _{g \in G} f(g) \quad\left(f \in \ell^{\infty}(G)\right)
$$

is a strictly increasing left invariant submean on $\ell^{\infty}(G)$.

Example 2. If there is a nonempty $S_{0} \subset S$ such that $s S_{0} \supset S_{0}$ for each $s \in S$, then

$$
\mu_{0}(f)=\sup _{s \in S_{0}} f(s) \quad\left(f \in \ell^{\infty}(S)\right)
$$

defines a strictly increasing left subinvariant submean on $\ell^{\infty}(S)$. In particular, if $S$ has a right zero $s_{0}$ so that $s s_{0}=s_{0}$ for all $s \in S$, then $\mu_{0}(f)=f\left(s_{0}\right)$ is a strictly increasing left invariant submean on $\ell^{\infty}(S)$. More generally, if $S$ has a left ideal $S_{0}=G_{0}$ which is a group, then $\mu_{0}$ defined above is a strictly increasing left invariant submean on $\ell^{\infty}(S)$.

Example 3. Let $S$ be a left reversible semitopological semigroup and let $\Gamma$ be the collection of all closed right ideals of $S$. Given any submean $\nu$ on a left invariant, positively semilinear subset $X$ of $L U C(S)$ that contains positive constants, we define

$$
\mu(f)=\inf _{J \in \Gamma} \sup _{s \in J} \nu\left(l_{s} f\right) \quad(f \in X) .
$$

Then $\mu$ is a left subinvariant submean on $X$. If $\nu$ is strictly increasing, then so is $\mu$. 
As a special case, for discrete $S$ we can take the submean $\nu$ on $\ell^{\infty}(S)$ defined by $\nu(f)=\sup _{s \in S} f(s)$. Then $\sup _{s \in J} \nu\left(l_{s} f\right)=\sup _{s \in J} f(s)$, and so

$$
\mu(f)=\inf _{J \in \Gamma} \sup _{s \in J} f(s) \quad\left(f \in \ell^{\infty}(S)\right)
$$

defines a strictly increasing left subinvariant submean on $\ell^{\infty}(S)$.

A submean $\mu$ on $\ell^{\infty}(S)$ is called supremum admissible if for any bounded family $\left\{f_{\alpha}: \alpha \in \Delta\right\} \subset \ell^{\infty}(S)$

$$
\mu\left(\sup _{\alpha \in \Delta} f_{\alpha}\right)=\sup _{\alpha \in \Delta} \mu\left(f_{\alpha}\right)
$$

where $\sup _{\alpha \in \Delta} f_{\alpha} \in \ell^{\infty}(S)$ is defined by $\left(\sup _{\alpha \in \Delta} f_{\alpha}\right)(s)=\sup _{\alpha \in \Delta}\left(f_{\alpha}(s)\right)$ $(s \in S)$.

The submeans defined in Examples 1 and 2]are supremum admissible. If $S$ is a finite semigroup, then every submean on $\ell^{\infty}(S)$ is supremum admissible.

The following was proved in [59].

Theorem 6.1. Let $S$ be a semigroup that acts on a weak* closed convex set $K \neq \emptyset$ of a dual Banach space $E=\left(E_{*}\right)^{*}$ as norm nonexpansive self mappings. Suppose that $\ell^{\infty}(S)$ has a strictly increasing supremum admissible left subinvariant submean. If $K$ has normal structure and there is $c \in K$ such that $\left\{T_{s} c: s \in S\right\}$ is bounded, then $K$ has a common fixed point for $S$.

For $S=G$ being a group, since $\ell^{\infty}(G)$ always has a strictly increasing left invariant submean (Example 1) that is supremum admissible we immediately get the following result.

Corollary 6.2. Let $G$ be a group that acts on a weak* closed convex set $K \neq \emptyset$ of a dual Banach space $E=\left(E_{*}\right)^{*}$ as norm nonexpansive self mappings. If $K$ has normal structure and there is $c \in K$ such that $\left\{T_{g} c: g \in G\right\}$ is bounded, then $K$ has a common fixed point for $G$.

Regarding actions on a weak* compact convex subset of a dual Banach space, the following were obtained in [59].

Theorem 6.3. Let $S$ be a semitopological semigroup and $\mathcal{S}=\left\{T_{s}: s \in\right.$ $S\}$ be a norm nonexpansive representation of $S$ on a nonempty weak ${ }^{*}$ compact convex subset $K$ of a dual Banach space $E=\left(E_{*}\right)^{*}$. Suppose that $K$ has the normal structure. Then $K$ contains a common fixed point for $S$ if one of the following conditions holds.

(1) $S$ is left reversible and the representation is weak* continuous; 
(2) $A P(S)$ has a LIM and the representation is separately continuous and equicontinuous when $K$ is equipped with the weak* topology of $E$;

(3) $L U C(S)$ has a LIM and the mapping $(s, x) \mapsto T_{s} x$ from $S \times K$ into $K$ is jointly continuous when $K$ is equipped with the weak* topology of $E$;

(4) $R U C(S)$ has a LIM and the representation is separately continuous and equicontinuous when $K$ is equipped with the weak ${ }^{*}$ topology of E.

\section{SOME OPEN PROBLEMS}

We conclude this paper with several open questions as follows.

Open Problem 2. Does a semitopological semigroup $S$ have the fpp $\left(F_{*}\right)$ if $L U C(S)$ has a LIM?

The question is open even for discrete case [40]. It was shown in [56, Proposition 6.1] that a weak version of property $\left(F_{*}\right)$ holds if $L U C(S)$ has a LIM.

Related to Problem 2, the following extension of [52, Theorem 5.3] were proved in [56].

Theorem 7.1. If $S$ is a left reversible or left amenable semitopological semigroup, then the following fixed point property holds.

$\left(F_{* s}\right):$ Whenever $\mathcal{S}=\left\{T_{s}: s \in S\right\}$ is a norm nonexpansive representation of $S$ on a nonempty norm separable weak* compact convex set $C$ of the dual space of a Banach space $E$ and the mapping $(s, x) \mapsto T_{s}(x)$ from $S \times C$ to $C$ is jointly continuous when $C$ is endowed with the weak* topology of $E^{*}$, then there is a common fixed point for $S$ in $C$.

Open Problem 3. Let $S$ be a (discrete) semigroup. If the fpp $\left(F_{* s}\right)$ holds, does $W A P(S)$ have a LIM? We also do not know whether the existence of a LIM on $W A P(S)$ is sufficient to ensure the fpp $\left(F_{* s}\right)$.

A partial affirmative answer to Problem 3 was given in [56, Proposition 6.5], which we quote as follows.

Proposition 7.2. Suppose that $S$ has the fixed point property $\left(F_{* s}\right)$. Then

(1) $A P(S)$ has a LIM;

(2) $W A P(S)$ has a LIM if $S$ has a countable left ideal.

Consider partially bicyclic semigroups $S_{2}=\langle e, a, b, c| a b=e, a c=$ $e\rangle$ and $S_{1,1}=\langle e, a, b, c, d \mid a b=e, c d=e\rangle$. We know that they are not 
left amenable. So they do not have the fpp $\left(F_{*}\right)$. It is worth mentioning that $W A P\left(S_{2}\right)$ and $A P\left(S_{1,1}\right)$ both have a LIM (Theorem 2.6).

Open Problem 4. Does the partially bicyclic semigroup $S_{2}$ have the $\operatorname{fpp}\left(F_{* s}\right)$ ?

If the answer to the above question is yes, then, due to Theorem 2.6. $S$ having $\left(F_{* s}\right)$ is not equivalent to $S$ being left reversible; if the answer is no, then the converse of Proposition 7.2(2) does not hold even for a countable semigroup $S$.

Theorem 2.5]improves [55, Theorem 3.8] by removing the separability condition on $S$ assumed there. We wonder whether the same thing can be done to Theorem 2.1 .

Open Problem 5. Does fpp (F) hold for a semitopological semigroup $S$ if $W A P(S)$ has a LIM?

If $S$ is a locally compact group, then $S$ is extremely left amenable only when $S$ is a singleton [23]. However, a non-trivial topological group which is not locally compact can be extremely left amenable. In fact, let $S$ be the group of unitary operators on an infinite dimensional Hilbert space with the strong operator topology, then $S$ is extremely left amenable [25]; Theorem 4.5] shows that an F-algebra $A$ is left amenable if and only if the semigroup of normal positive functions of norm 1 on $A^{*}$ is extremely left amenable. For more examples we refer to [47].

Open Problem 6. Suppose that $S$ is extremely left amenable and $C$ is a weakly closed subset of a Banach space $E$, and suppose that $\mathcal{S}$ is a weakly continuous and norm nonexpansive representation of $S$ on $C$ such that $\left\{T_{s} c: s \in S\right\}$ is relatively weakly compact for some $c \in C$. Does $C$ contain a fixed point for $S$ ?

We know that the answer is "yes" when $S$ is discrete. Indeed, in this case, for each finite subset $\sigma$ of $S$ there is $s_{\sigma} \in S$ such that $s s_{\sigma}=s_{\sigma}$ for all $s \in \sigma$ by a theorem of Granirer's [22] (see also [56, Theorem 4.2] for a short proof). Consider the net $\left\{s_{\sigma} c\right\}$. By the relative weak compactness of $S c$, there is $z \in \overline{S c}^{\text {wk }} \subset C$ such that (go to a subnet if necessary) wk- $\lim _{\sigma} s_{\sigma} c=z$. Then, as readily checked, $T_{s} z=z$ for all $s \in S$ by the weak continuity of the $S$ action on $C$.

More generally, the answer to Problem [6 is still affirmative (even without the norm nonexpansiveness assumption) if the representation is jointly continuous when $C$ is equipped with the weak topology of $E$. This is indeed a consequence of Theorem 2.9(a).

Open Problem 7. Let $C$ be a nonempty closed convex subset of the sequence space $c_{0}$ and $\mathcal{S}$ be a representation of a commutative 
semigroup $S$ as nonexpansive mappings on $C$. Suppose that $\left\{T_{s} c: s \in\right.$ $S\}$ is relatively weakly compact for some $c \in C$. Is $F(\mathcal{S}) \neq \emptyset$ ?

One may not drop the relative weak compactness condition on the orbit of $c$. For example, on the unit ball of $c_{0}$ define $T\left(\left(x_{i}\right)\right)=$ $\left(1, x_{1}, x_{2}, \cdots\right)$. Then $T$ is nonexpansive, and obviously $T$ has no fixed point in the unit ball.

Open Problem 8. Let $A$ be an F-algebra. Let $\left(F_{n}^{\prime}\right)$ denote the same property as $\left(F_{n}\right)$ with "jointly continuous" replaced by "separately continuous" on compact subsets of $E$. Does $\left(F_{n}^{\prime}\right)$ imply $\left(F_{n}\right)$ ?

Regard the F-algebra $A$ as the Banach $A$-bimodule with the module multiplications given by the product of $A$. Then the dual space $A^{*}$ is a Banach $A$-bimodule. We say that a subspace $X$ of $A^{*}$ is topologically left (resp. right) invariant if $a \cdot X \subset X$ (resp. $X \cdot a \subset X)$ for each $a \in A$; We call $X$ topologically invariant if it is both left and right topological invariant. An element of $A^{*}$ is almost periodic (resp. weakly almost periodic) if the map $a \mapsto f \cdot a$ from $A$ into $A^{*}$ is a compact (resp. weakly compact) operator. Let $A P(A)$ and $W A P(A)$ denote the collection of almost periodic and weakly almost periodic functions on $A$ respectively. Then $A P(A)$ and $W A P(A)$ are closed topologically invariant subspaces of $A^{*}$. Furthermore, $1 \in A P(A) \subset W A P(A)$. When $G$ is a locally compact group and $A=L^{1}(G)$, then $A P(A)=$ $A P(G)$ and $W A P(A)=W A P(G)$.

Let $\left(F_{n}^{A}\right)$ denote the same property as $\left(F_{n}\right)$ with joint continuity replaced by equicontinuity on compact subsets of $E$. It is known that if $A$ satisfies $\left(F_{n}^{A}\right)$ then $A P(A)$ has a TLIM (see [41]).

Open Problem 9. Does the existence of TLIM on $A P(A)$ imply $\left(F_{n}^{A}\right)$ for all $n \geq 1$ ?

Let $\left(F_{n}^{W}\right)$ denote the same property $\left(F_{n}^{A}\right)$ with equicontinuity on compact subsets of $E$ replaced by quasi-equicontinuity on compact subsets of $E$ (which means the closure of $S$ in the product space $E^{K}$, for each compact set $K \subset E$, consists only of continuous maps from $K$ to $E)$. We have known that if $A$ satisfies $\left(F_{n}^{W}\right)$ for each $n \geq 1$ then $W A P(A)$ has a TLIM (see [41]).

Open Problem 10. Does the existence of TLIM on $W A P(A)$ imply $\left(F_{n}^{W}\right)$ for all $n \geq 1$ ?

Open Problem 11. Can separability of the semitopological semigroup in Theorem 5.5 be dropped? 


\section{REFERENCES}

[1] D. Alspach, A fixed point free nonexpansive map, Proc. Amer. Math. Soc. 82 (1981), 423-424.

[2] S. Atsushiba and W. Takahashi, Nonlinear ergodic theorems without convexity for nonexpansive semigroups in Hilbert spaces, J. Nonlinear Convex Analysis, 14 (2013), 209-219.

[3] U. Bader, T. Gelander and N. Monod, A fixed point theorem for $L^{1}$ spaces, Invent. Math. 189 (2012), 143-148.

[4] E. Bédos and L. Tuset, Amenability and co-amenability for locally compact quantum groups, Internat. J. Math. 14 (2003), 865-884.

[5] T. D. Benavides and M. A. J. Pineda, Fixed points of nonexpansive mappings in spaces of continuous functions, Proc. Amer. Math. Soc. 133 (2005), 30373046 .

[6] T. D. Benavides, M. A. J. Pineda and S. Prus, Weak compactness and fixed point property for affine mappings, J Funct. Anal. 209 (2004), 1-15.

[7] F. E. Browder, Non-expansive nonlinear operators in Banach spaces, Proc. Nat. Acad. Sci. U. S. A., 54 (1965), 1041-1044.

[8] R. E. Bruck, On the center of a convex set, Dokl. Akad. Nauk SSSR (N. S.), 59 (1948), 837-840.

[9] A.V. Buhvalov, G.Ja. Lozanovskii, On sets closed in measure measure in spaces of measurable functions, Trans. Moscow Math. Soc. 2 (1978), 127-148.

[10] H. G. Dales, A. T.-M. Lau and D. Strauss, Second duals of measure algebras, Dissertations Math 481, 2012.

[11] S. Desaulniers, R. Nasr-Isfahani and M. Nemati, Common fixed point properties and amenability of a class of Banach algebras, J. Math. Anal. Appl. 402 (2013), 536-544.

[12] P.G. Dodds, T.K. Dodds, F.A. Sukochev and O.Ye. Tikhonov, A noncommutative Yosida-Hewitt theorem and convex sets of measurable operators closed locally in measure, Positivity 9 (2005), 457-484.

[13] P. N. Dowling, C. J. Lennard and B. Turett, Weak compactness is equivalent to the fixed point property in $c_{0}$, Proc. Amer. Math. Soc. 132 (2004), 1659-1666

[14] J. Duncan and I. Namioka, Amenability of inverse semigroups and their semigroup algebras, Proc. Roy. Soc. Edinburgh Sect A 80 (1978), 309-321.

[15] J. Duncan and A. L. T. Paterson, Amenability for discrete convolution semigroup algebras, Math. Scand. 66 (1990), 141-146.

[16] P. Eymard, Sur les applications qui laissent stable l'ensemble des fonctions presque-periodiques, Bull. Soc. Math. France 89 (1961) 207-222.

[17] K. Fan, Invariant subspaces for a semigroup of linear operators, Nederl. Akad. Wetensch. Proc. Ser. A 68, Indag. Math. 27 (1965), 447-451.

[18] K. Fan, Invariant cross-sections and invariant linear subspaces, Israel J. Math. 2 (1964), 19-26.

[19] K. Fan, Invariant subspaces of certain linear operators, Bull. Amer. Math. Soc. 69 (1963), 773-777.

[20] K. Goebel and W. A. Kirk, Topics in metric fixed point theory, Cambridge Studies in Advanced Mathematics 28, 1990.

[21] K. Goebel and W. A. Kirk, Classical theory of nonexpansive mappings, Handbook of metric fixed point theory, Kluwer Acad. Publ., Dordrecht, 2001, 49-91. 
[22] E. Granirer, Extremely amenable semigroups, Math. Scand. 17 (1965), 177197.

[23] E. Granirer and A. T.-M. Lau, Invariant means on locally compact groups, Illinois J. Math. 15 (1971) 249-257

[24] F. P. Greenleaf, Invariant means on topological groups and their applications. Van Nostrand Mathematical Studies, No. 16 Van Nostrand Reinhold Co., New York-Toronto, Ont.-London, 1969.

[25] M. Gromov and V. D. Milman, A topological application of the isoperimetric inequality. Amer. J. Math. 105 (1983), 843-854.

[26] P. Harmand, D. Werner and W. Werner, M-ideals in Banach spaces and Banach algebras, Lecture Notes in Math., 1547, Springer-Verlag, 1993.

[27] R. D. Holmes and A. T.-M. Lau, Nonexpansive actions of topological semigroups and fixed points, J. Lond. Math. Soc. 5 (1972), 330-336.

[28] R. Hsu, Topics on weakly almost periodic functions, Ph.D. Thesis, SUNY at Buffalo, 1985.

[29] Z. Hu, M. Sangani Monfared and T. Traynor, On character amenable Banach algebras, Studia Math. 193 (2009), 53-78.

[30] E. Kaniuth, and A. T.M. Lau, Fourier and Fourier-Stieltjes algebras on locally compact groups, Mathematical Surveys and Monographs 231, AMS Providence, RI, 2018.

[31] P. Kocourek, W. Takahashi and J.-C. Yao, Fixed point theorems and weak convergence theorems for generalized hybrid mappings in Hilbert spaces, Taiwanese J. Math. 14 (2010), 2497-2511.

[32] I. S. Iohvidov, Unitary operators in a space with an indefinite metric, Zap. Mat. Otd. Fiz.-Mat. Fak. i Har'kov. Mat. Obsc. (4) 21 (1949), 79-86.

[33] I. S. Iohvidov and M. G. Krein, Spectral theory of operators in spaces with indefinite metric I, Amer. Math. Soc. Transl. (2) 13 (1960) 105-175.

[34] W. A. Kirk, A fixed point theorem for mappings which do not increase distances, American Math Monthly, 72 (1965), 1004-1006.

[35] M. G. Krein, On an application of the fixed-point principle in the theory of linear transformations of spaces with an indefinite metric, Amer. Math. Soc. Transl. (2) 1 (1955), 27-35.

[36] A. T.-M. Lau, Invariant means on almost periodic functions and fixed point properties, Rocky Mountain J of Math. 3 (1973), 69-76.

[37] A. T.-M. Lau, Some fixed point theorems and $\mathrm{W}^{*}$-algebras, Fixed point theory and applications (ed. S. Swaminathan) Academic Press, (1976), 121-129.

[38] A. T.-M. Lau, Semigroup of nonexpansive mappings on a Hilbert space, J. Math. Anal. Appl. 105 (1985), 514-522.

[39] A. T.-M. Lau, Amenability of semigroups, The analytic and topological theory of semigroups (ed. K. H. Hoffmann, J. D. Lawson, and J. S. Pym), de Gruyter, Berlin, 1990, 313-334.

[40] A. T.-M. Lau, Amenability and fixed point property for semigroup of Nonexpansive mappings, Fixed point theory and applications (ed. M. A. Thera and J. B. Baillon), Pitman Research Notes in Mathematical Series, 252 (1991), 303-313. 
[41] A. T.-M. Lau, Fourier and Fourier-Stieltjes algebras of a locally compact group and amenability, Topological vector spaces, algebras and related areas (Hamilton, ON, 1994), 79-92, Pitman Res. Notes Math. Ser. 316, Longman Sci. Tech., Harlow, 1994.

[42] A. T.-M. Lau, Analysis on a class of Banach algebras with applications to harmonic analysis on locally compact groups and semigroups, Fund. Math. 118 (1983), 161-175.

[43] A. T.-M. Lau, Finite-dimensional invariant subspaces for a semigroup of linear operators, J. Math. Anal. Appl. 97 (1983), 374-379.

[44] A. T.-M. Lau, Finite dimensional invariant subspace properties and amenability, J. Nonlinear Convex Anal. 11 (2010), 587-595.

[45] A. T.-M. Lau and M. Leinert, Fixed point property and the Fourier algebra of a locally compact group, Trans. Amer. Math. Soc. 360 (2008), 6389-6402. .

[46] A. T.-M. Lau and V. Losert, The $\mathrm{C}^{*}$-algebra generated by operators with compact support on a locally compact group, J. Funct. Anal. 112 (1993), $1-30$.

[47] A. T.-M. Lau and J. Ludwig, Fourier-Stieltjes algebra of a topological group, Adv. Math. 229 (2012), 2000-2023.

[48] A. T.-M. Lau and P. F. Mah, Normal structure in dual Banach spaces associated with a locally compact group, Trans. Amer. Math. Soc. 310 (1988), 341-353.

[49] A. T.-M. Lau, P, F. Mah and A. Ulger, Fixed point property and normal structure for Banach spaces associated to locally compact groups, Proc. Amer. Math. Soc. 125 (1997), 2021-2027.

[50] A. T.-M. Lau, A. L. T. Paterson and J. C. S. Wong, Invariant subspace theorems for amenable groups, Proc. Edinburgh Math. Soc. 32 (1989), 415-430.

[51] A. T.-M. Lau and J. C. S. Wong, Finite dimensional invariant subspaces for measurable semigroups of linear operators, J. Math. Anal. Appl. 127 (1987), 548-558.

[52] A. T.-M. Lau and W. Takahashi, Invariant means and fixed point properties for non-expansive representations of topological semigroups, Topological Methods in Nonlinear Analysis, J. Juliusz Schauder Center, 5 (1995), 39-57.

[53] A. T.-M. Lau and W. Takahashi, Invariant submeans and semigroups of nonexpansive mappings on Banach spaces with normal structure, J. Funct. Anal. 142 (1996), 79-88.

[54] A. T.-M. Lau and W. Takahashi, Nonlinear submeans on semigroups, Topl. Methods Nonlinear Anal., 22 (2003), 345-353.

[55] A. T.-M. Lau and Y, Zhang, Fixed point properties of semigroups of nonexpansive mappings, J. Funct. Anal. 254 (2008), 2534-2554.

[56] A. T.-M. Lau and Y. Zhang, Fixed point properties for semigroups of nonlinear mappings and amenability, J. Funct. Anal. 263 (2012), 2949-2677.

[57] A. T.-M. Lau and Y. Zhang, Finite dimensional invariant subspace property and amenability for a class of Banach algebras, Trans. Amer. Math. Soc., 368 (2016), 3755-3775.

[58] A. T.-M. Lau and Y. Zhang, Fixed point properties for semigroups of nonlinear mappings on unbounded sets, J. Math. Anal. Appl. 433 (2016), 1204-1209. 
[59] A. T.-M. Lau and Y. Zhang, Fixed point properties for semigroups of nonexpansive mappings on convex sets in dual Banach spaces, Studia. Math. 17 (2018), 67-87.

[60] C. Lennard, $C_{1}$ is uniformly Kadec-Klee, Proc. Amer. Math. Soc. 109 (1990), 71-77.

[61] T. C. Lim, Characterizations of normal structure, Proc. Amer. Math. Soc. 43 (1974), 313-319.

[62] T. C. Lim, Asymptotic centers and non-expansive mappings in some conjugate spaces, Pacific J. Math. 90 (1980), 135-143.

[63] V. Losert, The Derivation problem for group algebras, Annals of Math. 168 (2008), 221-246.

[64] B. Maurey, Points fixes des contractions de certains faiblement compacts de $L^{1}$, Seminaire d'Analyse Functionnelle 80-81, Ecole Polytech., Palaiseau, 1981.

[65] T. Mitchell, Fixed point of reversible semigroups of non-expansive mappings, Kodai Math. Seminar Rep. 22 (1970), 322-323.

[66] T. Mitchell, Topological semigroups and fixed points, Illinois J. Math. 14 (1970), 630-641.

[67] M. Sangani Monfared, Character amenability of Banach algebras, Math. Proc. Cambridge Philos. Soc. 144 (2008), 697-706.

[68] N. Mizoguchi and W. Takahashi, On the existence of fixed points and ergodic retractions for Lipshitzian semigroups in Hilbert spaces, Nonlinear Analysis 14 (1990), 69-80.

[69] M. A. Naimark, On commuting unitary operators in spaces with indefinite metric. Acta Sci. Math. (Szeged) 24 (1963) 177-189.

[70] M. A. Naimark, Commutative unitary permutation operators on a $\Pi_{k}$ space, Soviet Math. 4 (1963) 543-545.

[71] J.-P. Pier, Amenable locally compact groups, Pitman Research in Math. 172, Longman Group UK LImited, 1988.

[72] L. Pontrjagin, Hermitian operators in spaces with indefinite metric, Bull. Acad. Sci. URSS. Ser. Math. [Izvestia Akad. Nauk SSSR] 8 (1944) 243-280.

[73] W. O. Ray, The fixed point property and unbounded sets in Hilbert space, Trans, Amer. Math. Soc. 258 (1980), 531-537.

[74] Z.-J. Ruan, Amenability of Hopf von Neumann algebras and Kac Algebras, J. Funct. Anal. 139 (1996) 466-499.

[75] S. Sakai, C*-algebras and $\mathrm{W}^{*}$-algebras, Springer Verlag, 1971.

[76] M. Skantharajah, Amenable hypergroups. Illinois J. Math. 36 (1992) 15-46.

[77] B. Sims, Examples of fixed point free mappings, Handbook of metric fixed point theory, (ed. W. A. Kirk and B. Sims), Kluwer Acad. Publ., Dordrecht, 2001, $35-48$.

[78] W. Takahashi, Fixed point theorem for amenable semigroups of non-expansive mappings, Kodai Math. Sem Rep. 21 (1969), 383-386.

[79] W. Takahashi, N.-C. Wong and J.-C. Yao, Attractive point and mean convergence theorems for semigroups of mappings without continuity in Hilbert spaces, J. Fixed Point Theory Appl. 17 (2015), no. 2, 301-311.

[80] W. Takahashi and Y. Takeuchi, Nonlinear ergodic theorem without convexity for generalized hybrid mappings in a Hilbert space, J. Nonlinear Convex Anal. 12 (2011), 399-406. 
[81] D. Voiculescu, Amenability and Katz algebras, Algebres d'operateurs et leurs applications en physique mathematique (Proc. Colloq., Marseille, 1977), 451457, Colloq. Internat. CNRS, 274, CNRS, Paris, 1979.

[82] B. Willson, Invariant nets for amenable groups and hypergroups, Ph.D. thesis, University of Alberta, 2011.

[83] B. Willson, Configurations and invariant nets for amenable hypergroups and related algebras, Trans. Amer. Math. Soc., Trans. Amer. Math. Soc. 366 (2014), no. $10,5087-5112$.

$\dagger$ Department of Mathematical and Statistical sciences, University of Alberta, Edmonton, Alberta, T6G 2G1 Canada

E-mail address: anthonyt@ualberta.ca

$\ddagger$ Department of Mathematics, University of Manitoba, Winnipeg, Manitoba, R3T 2N2 CANAdA

E-mail address: yong.zhang@umanitoba.ca 